Mon. Not. R. Astron. Soc. 000,111(201X) Printed 5 September $2018 \quad$ (MN LATEX style file v2.2)

\title{
The efficiency of photodissociation for molecules in interstellar ices
}

\author{
J. Kalvāns ${ }^{\star}$ \\ Engineering Research Institute "Ventspils International Radio Astronomy Centre" of Ventspils University College, \\ Inženieru 101, Ventspils, LV-3601, Latvia
}

Accepted 2018 April 24. Received 2018 April 11; in original form 2017 December 30

\begin{abstract}
Processing by interstellar photons affects the composition of the icy mantles on interstellar grains. The rate of photodissociation in solids differs from that of molecules in the gas phase. The aim of this work was to determine an average, general ratio between photodissociation coefficients for molecules in ice and gas. A 1D astrochemical model was utilized to simulate the chemical composition for a line of sight through a collapsing interstellar cloud core, whose interstellar extinction changes with time. At different extinctions, the calculated column densities of icy carbon oxides and ammonia (relative to water ice) were compared to observations. The latter were taken from literature data of background stars sampling ices in molecular clouds. The bestfit value for the solid/gas photodissociation coefficient ratio was found to be $\approx 0.3$. In other words, gas-phase photodissociation rate coefficients have to be reduced by a factor of 0.3 before applying them to icy species. A crucial part of the model is a proper inclusion of cosmic-ray induced desorption. Observations sampling gas with total extinctions in excess of $\approx 22 \mathrm{mag}$ were found to be uncorrelated to modelling results, possibly because of grains being covered with non-polar molecules.
\end{abstract}

Key words: astrochemistry - molecular processes - stars: formation - ISM: clouds, molecules, cosmic rays

\section{INTRODUCTION}

Interstellar ices are formed by accretion and processing of chemical species on dust grains in dense parts of molecular clouds. Processes on grain surface and in the ice allow the formation of many species, whose synthesis in the gas phase is inefficient, e.g., molecular hydrogen, water, carbon dioxide $\mathrm{CO}_{2}$ and some complex organic molecules (COMs). The understanding of the chemical processing of interstellar ices is therefore essential in interpreting observations of dense clouds (and other objects) and clarifying the interstellar synthesis pathways of many chemical species.

The icy species are dissociated by the interstellar radiation field (ISRF) and cosmic-ray (CR) induced photons. The resulting molecular fragments may react with neighbouring molecules paving the way for a rich chemistry on the grain surface. These two steps - dissociation and subsequent reactions - constitute the phenomenon of photoprocessing of (interstellar) ices.

Ice photochemistry has a significant role in the synthesis of complex species in space (e.g., Öberg et al. 2011). The

\footnotetext{
* E-mail: juris.kalvans@venta.lv
}

most direct method in determining the effects of UV photons on the composition of astrophysical ices is astrochemical laboratory experiments. Irradiation increases the number of different chemical species even in pure ices consisting of simple molecules (Gerakines, Schutte \& Ehrenfreund 1996; Bossa et al. 2015; Martín-Doménech et al. 2015; Paardekooper, Bossa \& Linnartz 2016; Cruz-Diaz et al. 2018; Martín-Doménech. Cruz-Díaz \& Muñoz Caro 2018). Photolysis of icy mixtures considerably increases their chemical diversity Gerakines, Moore \& Hudson 2004; Öberg et al. 2009a, 2010; Islam, Baratta \& Palumbo 2014; Jiménez-Escobar et al. 2014; Muñoz Caro et al. 2014; Henderson \& Gudipati 2015; Fedoseev et al. 2016; Chuang et al. 2017).

The aim of this work is to evaluate the average efficiency of photodissociation for molecules in interstellar ices by comparing calculated and observed abundances of icy species. Such a study was permitted by the availability of observational data that relate the abundances of major icy species to total interstellar extinction $A_{\mathrm{V}}$ along a line of sight (LOS) towards a background star. By comparing the observed and calculated proportions of these species at certain $A_{\mathrm{V}}(\mathrm{LOS})$ values, it is possible to deduce, to what extent 
ice composition has been affected by the photoprocessing of the icy mantle.

The main tasks corresponding to the above-mentioned aim are developing an astrochemical model suitable for investigating photochemistry in interstellar ices and obtaining results that can be reliably compared with observational data. The macrophysical model is described in Section 2.1 while the chemical model is explained in Section 2.2 Section 2.3 is dedicated on the approach for evaluating the photodissociation efficiency for icy species. Section 3 describes the obtained results, with the final evaluation of the photodissociation efficiency discussed in Section 3.4.

\section{METHODS}

The chemical model 'Alchemic-Venta' Kalvāns 2015c) was employed. It is based on the tested ALCHEMIC engine (Semenov et al. 2010) and has been adapted for modelling contracting cloud cores. Attention was paid for improving model parts crucial to a ice photochemistry study with results compared to observations. These include the modelling of an entire LOS through the cloud core, newer data and more detailed description on photochemical processes in ice. The effects of CRs were revised in line with recent findings. In particular, cosmic-ray induced thermal desorption (CRD) plays a significant role in shaping the composition of the ices.

Because most of the observational data sample molecular cloud complexes, an embedded cloud core was considered. The observations (data and references listed in Appendix A) sample a range of column densities, which were represented in the model in the course of the time-dependent core contraction.

In order to compare observed and calculated composition of interstellar ices, we employ the abundances of icy species along LOS, relative to water ice. Such an abundance ratio allows for a more reliable comparison with observational data than using absolute abundances (or abundances relative to $\mathrm{H}_{2}$ ) because sight lines with similar $A_{\mathrm{V}}(\mathrm{LOS})$ may sample different amounts of dense gas and ices. This means, for example, that the observed relative abundance $X_{\text {obs,CO }}$ of solid carbon monoxide molecules is equal to the ratio of the observed $\mathrm{CO}$ ice and $\mathrm{H}_{2} \mathrm{O}$ ice column densities $N_{\mathrm{CO}} / N_{\mathrm{H}_{2} \mathrm{O}}$. If we employ column densities from the modelling results (at the respective $A_{\mathrm{V}}(\mathrm{LOS})$ ) in the latter relation, we obtain the calculated relative abundance of icy $\mathrm{CO}$

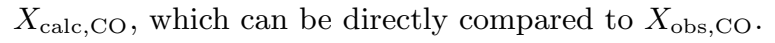

\subsection{Physical model}

In order to obtain $X_{\text {calc }}$, a chemical model of a contracting cloud core was employed. Relative abundances of icy species were calculated for a LOS that goes through the centre of the core. As the central density increases with time, $A_{\mathrm{V}}(\mathrm{LOS})$ rises, too. This allows to compare $X_{\text {obs }}$ and $X_{\text {calc }}$ at a range of $A_{\mathrm{V}}(\mathrm{LOS})$ values.

The macrophysical model was created to reflect an approximately feasible density structure and previous chemical evolution of ices for each LOS corresponding to the observed $A_{\mathrm{V}}(\mathrm{LOS})$ values in Table A1. Of course, such an approach is not universal because the observed cores may have different
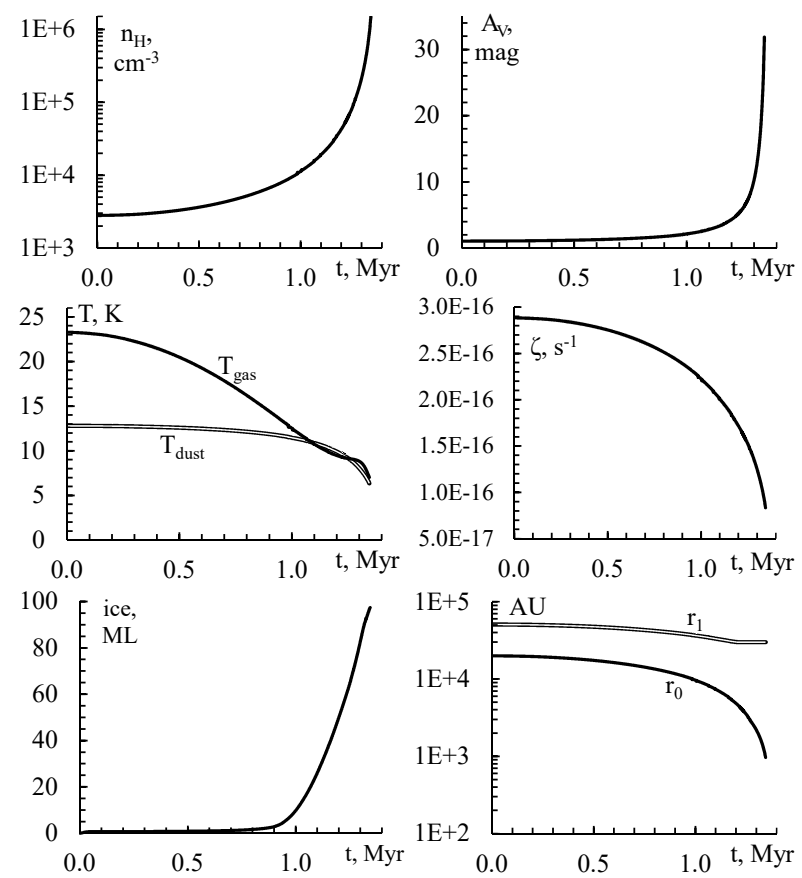

Figure 1. Evolution of key parameters at the centre of the modelled cloud core - density $n_{\mathrm{H}, 0}$, interstellar extinction $A_{\mathrm{V}}$, gas temperature $T_{\text {gas }}$, dust grain temperature $T_{\text {dust }}$, the CR ionization rate $\zeta$, indicative ice thickness (in monolayers) and cloud core parameters $r_{0}$ and $r_{1}$.

parameters and history. In the course of this study, we identified several objects from the observational data that likely do not fit the description employed in the model (Sections 3.1 and 3.2 .

The above means that the chemistry was calculated for a one-dimensional model of the cloud core. This is a significant improvement over our previous 0D point models, and allows for a more precise comparison with observational data towards background stars. We opted for a core evolution scenario where the central density of the core $n_{\mathrm{H}, 0}$ increases according to a delayed gravitational collapse. The cloud core starts with a $n_{\mathrm{H}, 0}=2800 \mathrm{~cm}^{-3}$, which grows according to Equation (1) of Neiad. Williams \& Charnlev (1990) until a maximum density of $1.5 \times 10^{6} \mathrm{~cm}^{-3}$ is reached in an integration time $t=1.39 \mathrm{Myr}$. This corresponds to $A_{\mathrm{V}}(\mathrm{LOS})$ from 2.1 to $63.7 \mathrm{mag}$. Higher central densities were not considered because the maximum extinction along LOS reported in observational data is 56 mag.

The considered core has to be representative to some extent to most of the dense gas clumps encountered in the interstellar clouds. Many of the high values of $A_{\mathrm{V}}(\mathrm{LOS})$ derived from the observations do not correspond to starless cores, indicating that these are heavily embedded or prestellar objects. Our chosen core can be described as a low-mass Bonnor-Ebert sphere (Ebert 1955; Bonnor 1956; McCrea 1957). The density profile for the sphere was expressed with a Plummer-like (Plummer 1911; Whitworth \& Ward-Thompson 2001) approximation:

$$
n_{\mathrm{H}, r}=n_{\mathrm{H}, 0}\left[1+\left(\frac{r}{2.88 r_{0}}\right)^{2}\right]^{-1.47}
$$


where $n_{\mathrm{H}, r}$ is density at a given radius $r$, and $r_{0}$ is the radius of the central density plateau. The density profile for each time step for the 1D model was calculated with this equation. We did not consider events that trigger the contraction of the core.

The physical parameters of the core $-n_{\mathrm{H}, 0}, r_{0}$, total (outer) radius $r_{1}$ and core mass $M_{\text {core }}=4.0 M_{\odot}$ - were adopted from the rather diffuse yet supercritical core No. 134 (hereinafter - \#134) from Table C.1 of Vavtet \& Haugbølle (2017). The increase of $n_{\mathrm{H}, 0}$ with time according to Nejad, Williams \& Charnley (1990) prompts changes in the core's density profile according to Equation (1). The central density $n_{\mathrm{H}, 0}=4.6 \times 10^{4} \mathrm{~cm}^{-3}$ corresponding to $\# 134$, is reached at integration time $t=$ 1.21 Myr.

The model follows the chemical evolution of gas parcels with a fixed mass coordinate along the LOS. In order to ensure that the absolute mass coordinate of each parcel is constant, the total mass of the core has to be constant. This was achieved by calculating the density plateau radius $r_{0}$ with a best-fit function in the form

$$
r_{0}=C_{1} n_{\mathrm{H}, 0} C_{2}
$$

where $C_{1}$ and $C_{2} \approx-1 / 2$ Keto \& Caselli 2010; Taquet, Charnley \& Sipilä 2014) are parameters adjusted so that $M_{\text {core }}$ always stays constant at $4.0 M_{\odot} . r_{0}$ varies from $20 \mathrm{kAU}$ at the start of the simulation to $970 \mathrm{AU}$ at the end. We note that the model does not employ the free-fall times calculated by Vavtet \& Haugbølle (2017) because the core is already contracting when its parameters reach those of \#134. Slight changes of the core outer radius $r_{1}$ were also permitted in order to ensure constant core mass. The evolution of $r_{0}$ and $r_{1}$ are shown graphically in the lower-right plot of Fig. 1

Our considered cloud core must not be isolated because the observations sample molecular cloud complexes. The core was assumed to be embedded in diffuse gas with isotropic column density $N_{\text {out }}=5 \times 10^{20} \mathrm{~cm}^{-3}$. This value is slightly higher than that characteristic to isolated starless cores (e.g., Lippok et al. 2013) because the considered core is part of a larger complex. $N_{\text {out }}$ cannot be much higher, otherwise the simulation would start with $A_{\mathrm{V}}$ (LOS) close to $3 \mathrm{mag}$, when an observable water ice layer should already be present on grain surfaces (Whittet et al. 2001).

Furthermore, we assume that the core resides in a planeparallel parent cloud that blocks the interstellar radiation from most directions reaching the sphere, i.e., the cloud core is irradiated only from two opposite sides along the LOS. This aspect is important when calculating the rates of ISRFdriven processes. Fig. 1 shows the evolution of the physical conditions and indicative ice thickness in the centre of the cloud core. The central part contributes significantly to the ice mass observed along the LOS.

Theoretical models show that CR ionization rate $\zeta$ in molecular clouds depends on their column density (Indriolo, Fields \& McCall 2009; Padovani, Galli \& Glassgold 2009; Chabot 2016). Here we calculated $\zeta$ according to the 'High' model of Ivlev et al. (2015). Following Padovani \& Galli (2013), $\zeta$ was modified by a factor of 0.3 to account for magnetic reflection of CRs at column densities relevant to this model. Fig. 1 shows that $\zeta$ values in the centre of the core change from $2.9 \times 10^{-16} \mathrm{~s}^{-1}$
Table 1. Initial abundances of chemical species relative to $\mathrm{H}$ nuclei.

\begin{tabular}{lc}
\hline Species & Abundance \\
\hline $\mathrm{H}_{2}$ & 0.5 \\
$\mathrm{He}$ & $9.00 \mathrm{E}-2$ \\
$\mathrm{C}$ & $1.40 \mathrm{E}-4$ \\
$\mathrm{~N}$ & $7.50 \mathrm{E}-5$ \\
$\mathrm{O}$ & $3.20 \mathrm{E}-4$ \\
$\mathrm{~F}$ & $6.68 \mathrm{E}-9$ \\
$\mathrm{Na}$ & $2.25 \mathrm{E}-9$ \\
$\mathrm{Mg}$ & $1.09 \mathrm{E}-8$ \\
$\mathrm{Si}$ & $9.74 \mathrm{E}-9$ \\
$\mathrm{P}$ & $2.16 \mathrm{E}-10$ \\
$\mathrm{~S}$ & $9.14 \mathrm{E}-8$ \\
$\mathrm{Cl}$ & $1.00 \mathrm{E}-9$ \\
$\mathrm{Fe}$ & $2.74 \mathrm{E}-9$ \\
\hline
\end{tabular}

to $8.3 \times 10^{-17} \mathrm{~s}^{-1}$. This approach is consistent with the CR energy spectrum used in calculating the whole-grain heating rate in Section 2.2.3 The flux of CR-induced photons $F_{\mathrm{CRph}}$ was modified proportionally, assuming that $F_{\mathrm{CRph}}=4875 \mathrm{~cm}^{-2} \mathrm{~s}^{-1}$ when $\zeta=1.3 \times 10^{-17} \mathrm{~s}^{-1}$ (Tomasko \& Spitzer 1968; Cecchi-Pestellini \& Aiello 1992).

A standard flux of $1.0 \times 10^{8} \mathrm{~cm}^{-2} \mathrm{~s}^{-1}$ for ISRF photons was assumed. Self- and mutual shielding of $\mathrm{H}_{2}, \mathrm{CO}$ and $\mathrm{N}_{2}$ with the help of the tabulated data of Lee et al. (1996) and Li et al. (2013) were included. The calculation of the gas temperature of the cloud was adopted from Kalvāns et al. (2017), while that of dust grains was calculated according to Hocuk et al. (2017).

The model considers olivine grains with radius $a=$ $0.1 \mu \mathrm{m}$ that constitute 1 per cent of cloud mass. The surface density of adsorption sites was taken to be $1.5 \times 10^{15} \mathrm{~cm}^{-2}$ (Hasegawa, Herbst \& Leung 1992). The assumed thickness of a single ice monolayer (ML) was $3.5 \times 10^{-8} \mathrm{~cm}$. Grain size and the number of surface adsorption sites $N_{s}$ are timedependent, taking into account the number of accreted ice MLs and the corresponding ice thickness.

\subsection{Chemical model}

\subsubsection{Chemical reactions network}

The modelled cloud core starts with hydrogen in molecular form and other elements in neutral atomic form with initial abundances listed in Table [1 For modelling gasphase chemical processes, the UMIST Database for Astrochemistry 2012 (UDfA12, McElroy et al. 2013) was employed. Photoreactions for icy species were also adopted from UDfA12. However, this database does not contain binary surface reactions. These were adopted from the COMs chemistry network of Garrod, Weaver \& Herbst (2008) with changes from Laas et al. (2011) and Kalvāns (2015b c). The energy barrier $E_{\mathrm{A}, \mathrm{O}+\mathrm{CO}}$ for the surface reaction $\mathrm{O}+$ $\mathrm{CO} \rightarrow \mathrm{CO}_{2}$, which is important for the production of $\mathrm{CO}_{2}$, was taken to be $630 \mathrm{~K}$ according to the experimental results of Minissale et al. (2013). $630 \mathrm{~K}$ also happens to be a best-fit value for $E_{\mathrm{A}, \mathrm{O}+\mathrm{CO}}$, which allows to obtain results that are more consistent with observations than values used previously - $290 \mathrm{~K}$ (Roser et al. 2001) or 
$1000 \mathrm{~K}$ (D'Hendecourt, Allamandola \& Greenberg 1985, see also Garrod \& Paulv 2011).

Surface binary reactions were described with the modified rate equations method, as in the original ALCHEMIC code (Caselli et al. 2002; Garrod et al. 2009). We included the diffusion-reaction competition following Garrod \& Pauly (2011). Reaction and diffusion barriers can be overcome either by thermal hopping or quantum tunnelling, depending on which is faster.

The desorption energies $E_{\mathrm{D}}$ for surface species were adopted from the COMs network (Garrod \& Herbst 2006; Garrod, Weaver \& Herbst 2008). These authors derived $E_{\mathrm{D}}$ for molecules on water ice surface. Their $E_{\mathrm{D}}$ values roughly agree with experimental values for the species relevant in this study (e.g. Martín-Doménech et al. 2014; Favolle et al. 2016). However, for CO-dominated ices that appear late in the evolution of the cloud core, desorption energy and the associated binding energy (see below) can be notably lower, as indicated by thermal desorption of pure ices (Collings et al. 2004; Luna et al. 2017). This aspect was taken into account when comparing modelling results with observational data (Section 3.2).

\subsubsection{Model of the icy grain mantle}

The description of the icy grain mantle was adopted from Kalvāns (2015c). The mantle is formed on the grain surface via the adsorption of neutral species. The mantle was described as consisting of four layers - the surface and three bulk-ice layers. The sticking coefficient for $\mathrm{H}$ and $\mathrm{H}_{2}$ was adopted from Thi. Woitke \& Kamp (2010), while that of other species was taken to be unity. Species in the bulk ice were assumed to be bound with absorption energy $E_{\mathrm{B}}$ that is three times higher than the adsorption energy $E_{\mathrm{D}}$ for surface species.

Diffusion occurs between adjacent ice mantle layers. The diffusion energy for species within the mantle $E_{\mathrm{b}, \mathrm{m}}$ was taken to be three times higher than that of surface species $E_{\mathrm{b}, \mathrm{s}}$ Kalvāns (2015a). The rates of chemical reactions for molecules in the bulk-ice layers were calculated by assuming that the molecule vibrates in its ice lattice cell. Because the cell is small, the molecule in consideration approaches its neighbours (potential reaction partners) with its characteristic vibration frequency $\nu_{0}$. A reaction occurs once the vibrating molecule happens to overcome a proximity barrier $E_{\text {prox }}$ Kalvāns (2015c). This is opposed to the diffusion process over a significant surface area, which is the case of surface species (Hasegawa, Herbst \& Leung 1992).

The relations between energies related to icy molecular processes on grains are summarized in Table 2. We refer the reader to Kalvāns (2015a c) for more details, discussion and justification on the chemistry of the icy mantle.

A conservative value of 0.50 for the binding to desorption energy ratio was adopted, as in the original COMs surface chemistry network of Garrod \& Herbst (2006). Values such as $0.3,0.35,0.40,0.50,0.55$ and 0.77 have been used for the $E_{\mathrm{b}} / E_{\mathrm{D}}$ ratio (Hasegawa. Herbst \& Leung 1992; Garrod 2013; Garrod \& Paulv|2011; Garrod \& Herbst 2006; Vasvunin et al. 2017; Ruffle \& Herbst 2001b, respectively). Test calculations were performed with all these $E_{\mathrm{b}} / E_{\mathrm{D}}$ ratios, with the middle value of 0.50 yielding results that are most consistent with the 'valid' observations, summarized in Section 3.2

\subsubsection{Desorption mechanisms}

The model considers several desorption mechanisms that transfer molecules from the grain surface layer to the gas phase. These include evaporation, photodesorption by the ISRF and CR-induced photons, reactive desorption (Garrod, Wakelam \& Herbst 2007), and CRD. We also included the encounter desorption from Hincelin, Chang \& Herbst (2015). The latter ensures that $\mathrm{H}_{2}$ does not accumulate on grains at temperatures lower than $\approx 9 \mathrm{~K}$.

Kalvāns (2015c) found that a selective desorption mechanism helps removing the over-abundance of icy carbon oxides $\mathrm{CO}$ and $\mathrm{CO}_{2}$ in time-dependent astrochemical simulations. The efficiency of such a mechanism should be inversely dependent on the $E_{\mathrm{D}}$ of the surface species. It was suggested that indirect reactive desorption by $\mathrm{H}$ atoms combining on grain surface may provide such a mechanism. However, little data is available about this mechanism beyond theoretical estimates and it was not included in the present model. Instead, recent data indicates that this role can be played by CRD. The ability of energetic ions to induce desorption of icy species has been shown experimentally (e.g., Johnson et al. 1991; Dartois et al. 2015) and studied theoretically (e.g., Bringa \& Johnson 2004; Herbst \& Cuppen 2006). Such ions may induce also other processes in ices (Kalvāns 2015a, and references therein).

Chabot (2016) and Kalvāns (2016) showed that CRDinducing whole-grain heating events happen more often than previously thought (see Hasegawa \& Herbst 1993; Roberts et al. 2007). Moreover, CR intensity (and thus, frequency of grain heating) depends on the column density of gas (Padovani. Galli \& Glassgold 2009).

In this paper, the basic approach on CRD was retained from Equation (15) of Hasegawa \& Herbst (1993), where it was assumed that CRD occurs for grains that are heated to $70 \mathrm{~K}$ for a cooling time $t_{\text {cool }}=10^{-5} \mathrm{~s}$. However, the frequency $f_{70}$ of grain encounters with CRs heating the grains to $70 \mathrm{~K}$ was recalculated using the data and methods of Kalvāns (2016) as explained in Appendix B Therefore, the CR-induced desorption rate coefficient is

$$
k_{\text {crd }}(i)=k_{\text {evap }}(i, 70 \mathrm{~K}) t_{\text {cool }}(70 \mathrm{~K}) \times f_{70}, s^{-1},
$$

where $k_{\text {evap }},(i, 70 \mathrm{~K}), \mathrm{s}^{-1}$ is the evaporation rate coefficient for species $i$ at $70 \mathrm{~K}$ and $t_{\text {cool }}(70 \mathrm{~K}) \times f_{70}$ is the 'duty cycle' or fraction of time spent in the vicinity of $70 \mathrm{~K}$ for the grain. The frequency of CRD events $f_{70}$ is defined with Equations (B1) and (B2) in Appendix B

\subsubsection{Photoprocessing of icy species}

The model considers ISRF and CR-induced photodissociation of species in all ice layers. The rate coefficients were taken from the corresponding gas-phase reactions and modified according to

$$
k_{\mathrm{ph}}^{\prime}=\frac{1}{2} \epsilon_{\mathrm{ph}} k_{\mathrm{ph}}\left(1-P_{\mathrm{abs}}\right)^{B}, s^{-1}
$$


Table 2. Summary of the energies characterizing the mobility icy species on the surface and in the bulk ice.

\begin{tabular}{llclll}
\hline \multirow{2}{*}{ Surface } & & \multicolumn{3}{c}{ Bulk ice (mantle sublayers) } \\
& Notation & Value & Energy & Notation & Value \\
\hline Desorption (adsorption) & $E_{\mathrm{D}}$ & (base data) $^{\mathrm{a}}$ & Absorption & $E_{\mathrm{B}}$ & $3.0 E_{\mathrm{D}}{ }^{\mathrm{c}, \mathrm{d}}$ \\
Binding (diffusion) & $E_{\mathrm{b}, \mathrm{s}}$ & $0.5 E_{D} \mathrm{~b}^{\mathrm{b}}$ & Binding (diffusion) & $E_{\mathrm{b}, \mathrm{m}}$ & $0.5 E_{\mathrm{B}}$ \\
$\ldots$ & $\cdots$ & $\cdots$ & Proximity & $E_{\mathrm{prox}}$ & $0.1 E_{\mathrm{B}} \mathrm{d}$ \\
\hline
\end{tabular}

${ }^{a}$ Surface chemistry network of Garrod. Weaver \& Herbst (2008); see text.

b Garrod \& Herbst (2006).

c Kalvāns (2015a).

d Kalvāns (2015c).

Table 3. Adopted total desorption yields for surface chemical species in the model.

\begin{tabular}{lccl}
\hline Molecule & $Y_{\text {ISRF }}$ & $Y_{\text {CRph }}$ & References \\
\hline $\mathrm{N}_{2}$ & $5.5 \mathrm{E}-03$ & $3.0 \mathrm{E}-03$ & $(1)$ \\
$\mathrm{O}_{2}$ & $9.3 \mathrm{E}-04$ & $9.3 \mathrm{E}-04$ & $(2)$ \\
$\mathrm{CO}$ & $5.7 \mathrm{E}-03$ & $3.9 \mathrm{E}-03$ & $(1)$ \\
$\mathrm{CH}_{4}$ & $2.0 \mathrm{E}-03$ & $2.2 \mathrm{E}-03$ & $(3)$ \\
$\mathrm{CO}_{2}$ & $f(B)^{*}$ & $f(B)^{*}$ & $(4)$ \\
$\mathrm{CH}_{3} \mathrm{OH}$ & $2.3 \mathrm{E}-04$ & $2.5 \mathrm{E}-04$ & $(5)$ \\
$\mathrm{H}_{2} \mathrm{O}$ & $f\left(B, T_{\text {dust }}\right)^{*}$ & $f\left(B, T_{\text {dust }}\right)^{*}$ & $(6)$ \\
$\mathrm{NH}_{3}$ & $2.0 \mathrm{E}-03$ & $0.0 \mathrm{E}-03$ & $(7)$ \\
Other & $1.0 \mathrm{E}-03$ & $1.0 \mathrm{E}-03$ & $\cdots$ \\
\hline
\end{tabular}

* Function of ice thickness $B(\mathrm{ML})$ and grain temperature $T_{\text {dust }}$.

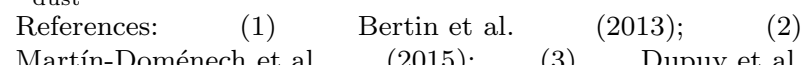
Martín-Doménech ët (2017): (4) Öberg, van Dishoeck \& Linnartz (2009c); (5) Bertin et al. (2016); (6) Öberg et al. (2009b); (7) Martín-Doménech. Cruz-Díaz \& Muñoz Caro (2018).

where $k_{\text {ph }}^{\prime}$ is the actual rate coefficient used in the model, $k_{\mathrm{ph}}$ is the dissociation rate coefficient for gaseous species (Equations (3) and (4) in McElroy et al. 2013), $P_{\text {abs }}$ is photon absorption probability per ice ML and $B$ number of ice MLs between the outer ice mantle surface and the molecule in consideration. The efficiency of photodissociation for icy species $\epsilon_{\mathrm{ph}}$ is defined with Equation (6) below. The factor $1 / 2$ was added to reflect the fact that a molecule residing on grain surface is partially shielded from incident radiation by the body of the grain, much larger than the molecule.

The value for $P_{\text {abs }}=0.007$ was derived from molecular dynamics simulations (Andersson \& van Dishoeck 2008). This means that, for a 100 ML thick mantle, molecules in the bottom layer (adjacent to grain nucleus) would be dissociated with a rate reduced by a factor of $\approx 0.5$. The $P_{\text {abs }}$ value of 0.007 can be compared to values derived from experimental dissociation cross-sections of water, where $P_{\text {abs }} \approx 0.002$ (Mason et al. 2006; Cruz-Diaz et al. 2014a). The higher, theoretically obtained value of $P_{\text {abs }}$ was employed here to account for photon losses in processes other than full molecular dissociation, which is the process measured in the experiments.

Experiments show that photodissociation and desorption (photodissociative desorption, PDD) of molecules in the surface layer occur simultaneously (Fillion et al. 2014; Bertin et al. 2016; Cruz-Diaz et al. 2016; Martín-Doménech. Muñoz Caro \& Cruz-Díaz 2016). In the case of amorphous water ice surface photodis- sociation, the proportion of water molecules going to the gas phase is about three per cent. A significant fraction of these desorbed molecules are dissociated (Andersson \& van Dishoeck 2008; Arasa et al. 2015). In astrochemical modelling, PDD has been accounted by Taquet et al. (2013) for the water molecule. In order to consider PDD at least to some extent for all surface molecules, we employed a general approach on PDD, which is described in Appendix C

The total rate coefficient for photodesorption (intact molecules plus their dissociated fragments) is calculated with

$$
k_{\mathrm{pd}}=\frac{1}{2} \times \frac{\sigma_{\mathrm{g}} F_{\mathrm{ph}} Y_{\mathrm{ph}}}{N_{s}}, \mathrm{~s}^{-1}
$$

where $\sigma_{\mathrm{g}}\left(\mathrm{cm}^{2}\right)$ is the grain cross section, $N_{s}$ is the number of adsorption sites on grain surface, $F_{\mathrm{ph}}$ is the flux $\left(\mathrm{s}^{-1} \mathrm{~cm}^{-2}\right)$ of either ISRF or CR-induced photons, and $Y_{\mathrm{ph}}$ is the total desorption yield either for interstellar $\left(Y_{\mathrm{ISRF}}\right)$ or CR-induced photons $\left(Y_{\mathrm{CRph}}\right)$.

Experimentally determined total desorption yields were adopted for $\mathrm{CO}, \mathrm{CH}_{4}, \mathrm{CO}_{2}, \mathrm{CH}_{3} \mathrm{OH}, \mathrm{N}_{2}, \mathrm{NH}_{3}, \mathrm{O}_{2}$ and $\mathrm{H}_{2} \mathrm{O}$. For other surface species, $Y_{\text {ph }}$ was taken to be $1.0 \times 10^{-3}$. The adopted values of the total yields, summarized in Table 3 . include both, intact molecule desorption and desorption of photodissociation fragments. There is a variety of available experimental photodesorption yields for some common icy species, such as carbon monoxide (Öberg et al. 2007; Öberg, van Dishoeck \& Linnartz 2009c; Muñoz Caro et al. 2010, 2016; Favolle et al. 2011b; Bertin et al. 2013; Chen et al. 2014; Paardekooper, Bossa \& Linnartz 2016), carbon dioxide (Öberg, van Dishoeck \& Linnartz 2009c; Fillion et al. 2014; Martín-Doménech et al. 2015), methanol (Öberg et al. 2009a; Bertin et al. 2016; Cruz-Diaz et al. 2016; Martín-Doménech. Muñoz Caro \& Cruz-Díaz 2016) and nitrogen (Öberg, van Dishoeck \& Linnartz 2009c; Bertin et al. 2013; Favolle et al. 2013). Whenever possible, we employed $Y_{\mathrm{ph}}$ values from experiments with mixed ices, which presumably more closely represent the actual icy mantles in the ISM.

\subsection{Investigating ice photodissociation efficiency}

The model described above was used to obtain results to be compared with abundances of icy species along observed LOS $\left(X_{\text {calc }}\right.$ and $\left.X_{\text {obs }}\right)$. We remind that at the core of this study is estimating the molecular photodissociation rate in interstellar ices. Photoprocessing of surface species was introduced in astrochemical modelling by Ruffle \& Herbst 
(2001a). They assumed that dissociation of icy species occurs with an efficiency equal to that of gas-phase species. This is also the approach currently used in most astrochemical models considering surface chemistry.

Some of current astrochemical models that consider layered ices do not apply photoprocessing for bulk-ice molecules, often because of computational reasons (e.g. Taquet. Charnlev \& Sipilä 2014; Ruaud. Wakelam \& Hersant 2016; Sipilä, Caselli \& Taquet 2016; Furuva et al. 2017). Others include full photoprocessing of the whole ice mantle (Chang \& Herbst 2016; Kalvāns et al. 2017; Garrod et al. 2017; Vasyunin et al. 2017). In this paper, we assume that photodissociation occurs with similar efficiency for surface and bulk molecules. However, for this core collapse model, the photodissociation of molecules in the surface layer is what affects most the calculated column densities of icy species. The photoprocess in the model is dominated by the ISRF photons, whose flux is much higher than that of CR-induced photons for most of the integration (and ice formation) time.

Following Mason et al. (2006) and Furuya et al. (2013), the ice molecule photodissociation efficiency $\epsilon_{\mathrm{ph}}$ can be expressed with

$$
\epsilon_{\mathrm{ph}}=\sigma_{\mathrm{ph}, \mathrm{ice}, i} / \sigma_{\mathrm{ph}, \text { gas }, i}
$$

where $\sigma_{\mathrm{ph}, \text { ice, } i}$ is the photodissociation cross section for a given species $i$ in solid phase and $\sigma_{\mathrm{ph}, \mathrm{gas}, i}$ is the cross section for the same species in the gas phase, usually a known quantity.

An assortment of experimental data on the photodissociation cross sections of species in interstellar ice analogues are available. We reviewed the data provided by Gerakines. Schutte \& Ehrenfreund (1996); Cottin, Moore \& Bénilan (2003); Mason et al. (2006); Öberg et al. (2009a, 2010) and Cruz-Diaz et al. (2014a b, 2016). Some of these data have been summarized in Table 1 of Oberg (2016). These experimental photodissociation cross sections were compared to ISRF photon cross sections of the respective gas-phase species derived from the UDfA12 database. It was found that, for most cases, the efficiency $\epsilon_{\mathrm{ph}}$ lies in the range of $0.03 \ldots 0.72$.

The above-mentioned experiments employ a variety of set-up approaches - temperature of ice freezing and during irradiation, pure ices or mixtures, substrate materials, irradiation UV spectra, quantification techniques of chemical species. In some cases, different experiments considering the same species (e.g., $\mathrm{CO}, \mathrm{CH}_{4}$ ) yield cross sections that differ by up to two orders of magnitude. Because of this, the most reasonable approach in this study was to adopt a single $\epsilon_{\mathrm{ph}}$ value for all photodissociation reactions. This approach also has the benefit of being simple and easily applicable in astrochemical models.

In order to obtain an estimate of $\epsilon_{\mathrm{ph}}$, we performed the 1D simulations with 14 values of $\epsilon_{\mathrm{ph}}$ that cover the range indicated by experiments (Table 4). For benchmarking, simulations with $\epsilon_{\mathrm{ph}}=0$ and $\epsilon_{\mathrm{ph}}=1$ were also included (a total of 1.6 million integration steps).

\section{RESULTS}

\subsection{Selection of observational data}

Several simple rules had to be obeyed when selecting the observational data. First, the observations have to sample quiescent medium, which means that the focus is on background stars. Second, the $A_{\mathrm{V}}(\mathrm{LOS})$ value must be derived; this means that a number of earlier observational studies were not useful for our purposes. Third, the column density (or abundance) of water ice must be known, so that it is possible to obtain $X_{\mathrm{obs}}$. The fourth and final aspect was that the column density of a second species, besides water, must be reported in the paper.

In total, we selected 77 sources (from 9 published papers) that conformed to the above rules. These are listed in Appendix A. Multiple observations sampling the same object were permitted (e.g., Elias 13, Elias 16). 18 of the sources are identified by authors (Shuping et al. 2000; Boogert et al. 2013) being in proximity of high-mass starforming regions, thus likely receiving irradiation doses significantly above to the average interstellar medium. This means that the model presented in Section 2 may be far from reflecting the conditions in these clouds. We included these irradiated samples in Appendix $\mathrm{A}$, figures and the ensuing discussion but they were not considered in deriving the final result of this investigation.

\subsection{Limitations of the calculated data}

In the numerical study, a single cloud core model was employed, while the observations sample multiple cores with basically unknown parameters. Because of this, it can be expected that not all observations can be used in this study. When comparing the observational data with the results of the calculations, it was realized that there exist two populations of observational data points that cannot be reproduced with the model.

The first set of such data points is sight lines with abundant interstellar solid methanol. This model is able to produce icy methanol with $X_{\text {calc, } \mathrm{CH}_{3} \mathrm{OH}}$ of 1.5 per cent at maximum (in simulation with $\epsilon_{\mathrm{ph}}=1.00$ ), while Whittet et al. (2011) report $X_{\mathrm{obs}, \mathrm{CH}_{3} \mathrm{OH}}$ of up to 12 per cent (Fig. 2). Such abundances can be reproduced for interstellar cloud cores that are stable and exist for hundreds of kyr (Kalvāns 2015b). Our modelled contracting prestellar core does not fit this description. Therefore, we assume that observations with $X_{\mathrm{obs}, \mathrm{CH}_{3} \mathrm{OH}}$ notably exceeding one per cent ( 5 per cent or more in this case) sample such long-lived cores and cannot be used for comparison with calculations. The respective data points are marked as red triangles in the figures.

The second data set, unsuitable for comparison with calculations, are observations sampling ices with $A_{\mathrm{V}}(\mathrm{LOS})$ values exceeding $\approx 22 \mathrm{mag}$. We were unable to achieve a good agreement for data points $\left(X_{\mathrm{obs}}\right.$ for $\mathrm{CO}, \mathrm{CO}_{2}$ and $\left.\mathrm{NH}_{3}\right)$ with $A_{\mathrm{V}}(\mathrm{LOS})$ exceeding this value within reasonable values for the parameters $E_{\mathrm{b}, \mathrm{s}} / E_{\mathrm{D}}, E_{\mathrm{A}, \mathrm{O}+\mathrm{CO}}$ and $\epsilon_{\mathrm{ph}}$. The reason for such a systemic deviation could be either that the parameters of the modelled cloud core deviate significantly from the observed objects, or, more likely, that the properties of the grain surface changes when the surface gets completely covered with $\mathrm{CO}$ and $\mathrm{N}_{2}$ as suggested by 


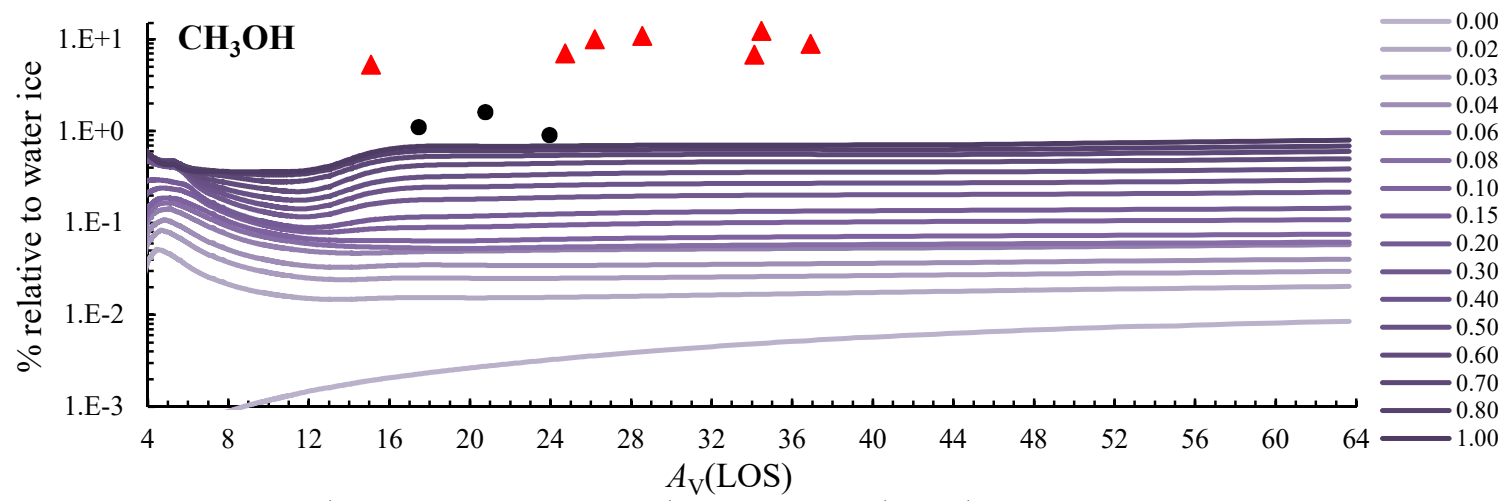

Figure 2. Comparison of calculated (curves with indicated $\epsilon_{\mathrm{ph}}$ ) and observed (circles) $\mathrm{CH}_{3} \mathrm{OH}: \mathrm{H}_{2} \mathrm{O}$ ice column density ratios as a function of $A_{r m V}(\mathrm{LOS})$. Red triangles $\left(\mathrm{CH}_{3} \mathrm{OH}: \mathrm{H}_{2} \mathrm{O}\right.$ exceeding 5 per cent) are suggested to represent observations of long-lived stable starless cloud cores.

Bergin, Langer \& Goldsmith (1995). Such a coverage with non-polar molecules reduces the adsorption and binding (diffusion) energies of surface species, affecting their chemistry. Easier diffusion of $\mathrm{CO}$ and other heavy reactants (e.g., O atoms) may allow them to be more competitive to $\mathrm{H}$ atoms, increasing the formation rate of $\mathrm{CO}_{2}$ and reducing that of $\mathrm{NH}_{3}$. This suggestion is based on simulations with lowered $E_{\mathrm{b}, \mathrm{s}} / E_{\mathrm{D}}<0.5$ ratios, mentioned in Section 2.2.2. A proper quantification and inclusion in the model of such a surface transformation is worth a separate study and was not attempted here. Cazaux et al. (2017) offer a recent experimental-theoretical study on this topic.

Summarizing the above, the following observational data points are excluded from estimating the value of $\epsilon_{\mathrm{ph}}$ in Section 3.4.

- observations sampling regions with increased irradiation from nearby massive stars, as indicated by the authors of the observational studies;

- observations associated with long-lived starless cores, judging by their high abundance of methanol ice;

- observations sampling regions with $A_{\mathrm{V}}$ (LOS) exceeding 22 mag.

Fig. 3 shows the modelled curves of $X_{\text {calc }}$ and $X_{\text {obs }}$ points from literature data for $\mathrm{CO}, \mathrm{CO}_{2}, \mathrm{NH}_{3}$ and $\mathrm{N}_{2}$. The observational data excluded from the evaluation of $\epsilon_{\mathrm{ph}}$ are indicated in the figure.

\subsection{Photochemistry of major interstellar icy species}

The most abundant observed interstellar icy species are water and carbon oxides. The most important photo-chemical process in ices in the interstellar medium (ISM) is the photo-production of icy $\mathrm{CO}_{2}$ at the expense of $\mathrm{CO}$ and $\mathrm{H}_{2} \mathrm{O}$ (Whittet et al. 1998). This study also investigated the effect of photoprocessing on the abundances of interstellar icy ammonia $\mathrm{NH}_{3}$ and methanol $\mathrm{CH}_{3} \mathrm{OH}$ (e.g., Boogert, Gerakines \& Whittet 2015).
While the choice of $\epsilon_{\mathrm{ph}}$ affects all icy species, there are only a few photoreactions, known and included in the network, that truly contribute to the abundances of the observed ice molecules. Before deriving the value of $\epsilon_{\mathrm{ph}}$, we briefly discuss the photochemistry of the main icy species.

The most abundant interstellar ice molecule is water, whose calculated and observed column density was used as a benchmark to obtain the relative abundances of $\mathrm{CO}$, $\mathrm{CO}_{2}$, and $\mathrm{NH}_{3}$. The main formation route of water is the hydrogenation of $\mathrm{O}$ atoms on grain surfaces. The dissociation of water is the most important ice photoreaction, for which $\epsilon_{\mathrm{ph}}$ we estimated to be 0.43 and 0.72 from the data of Mason et al. (2006) and Cruz-Diaz et al. (2014a), respectively. Photoprocessing tends to reduce the abundance of water ice by converting it to $\mathrm{O}_{2}, \mathrm{H}_{2} \mathrm{O}_{2}$ or, in the presence of $\mathrm{CO}$, to $\mathrm{CO}_{2}$ and $\mathrm{CH}_{3} \mathrm{OH}$. At least some the residual hydrogen escapes to the gas phase (Kalvāns \& Shmeld 2010; Cruz-Diaz et al. 2018). In addition to reduced dissociation rates, the UV absorption spectra is shifted for solid species when compared to gas. This was not explicitly considered in the study.

As a gas-phase product, carbon monoxide ice arises via accretion on grain surfaces. $\mathrm{CO}$ ice can be either hydrogenated to $\mathrm{CH}_{3} \mathrm{OH}$ or oxidized to $\mathrm{CO}_{2}$. For the conditions considered in this study, only the latter process is of significant importance. In ISM regions with a higher flux of UV radiation (empty circles in Fig. 3), the observed abundance of $\mathrm{CO}$ ice relative to water ice is notably lower than that obtained by the calculations. This agrees well with the picture that irradiation converts $\mathrm{CO}: \mathrm{H}_{2} \mathrm{O}$ icy mixtures into $\mathrm{CO}_{2}$.

Photochemistry is crucial for the formation of $\mathrm{CO}_{2}-$ when $\epsilon_{\mathrm{ph}}=0$, the calculated column density of $\mathrm{CO}_{2}$ relative to that of water ice is negligible, below 0.1 per cent. This means that all the $\mathrm{CO}_{2}$ in our model is produced via photoprocessing of surface species. $\mathrm{O}$ and $\mathrm{OH}$ radicals are split from $\mathrm{CO}$ and $\mathrm{H}_{2} \mathrm{O}$ before combining with $\mathrm{CO}$ molecules to produce $\mathrm{CO}_{2}$. Observational data shows that cloud cores under increased irradiation generally have a higher $\mathrm{CO}_{2}: \mathrm{H}_{2} \mathrm{O}$ ice abundance ratio, even exceeding 100 per cent. A similar effect can be observed for the long-lived cloud cores (identi- 

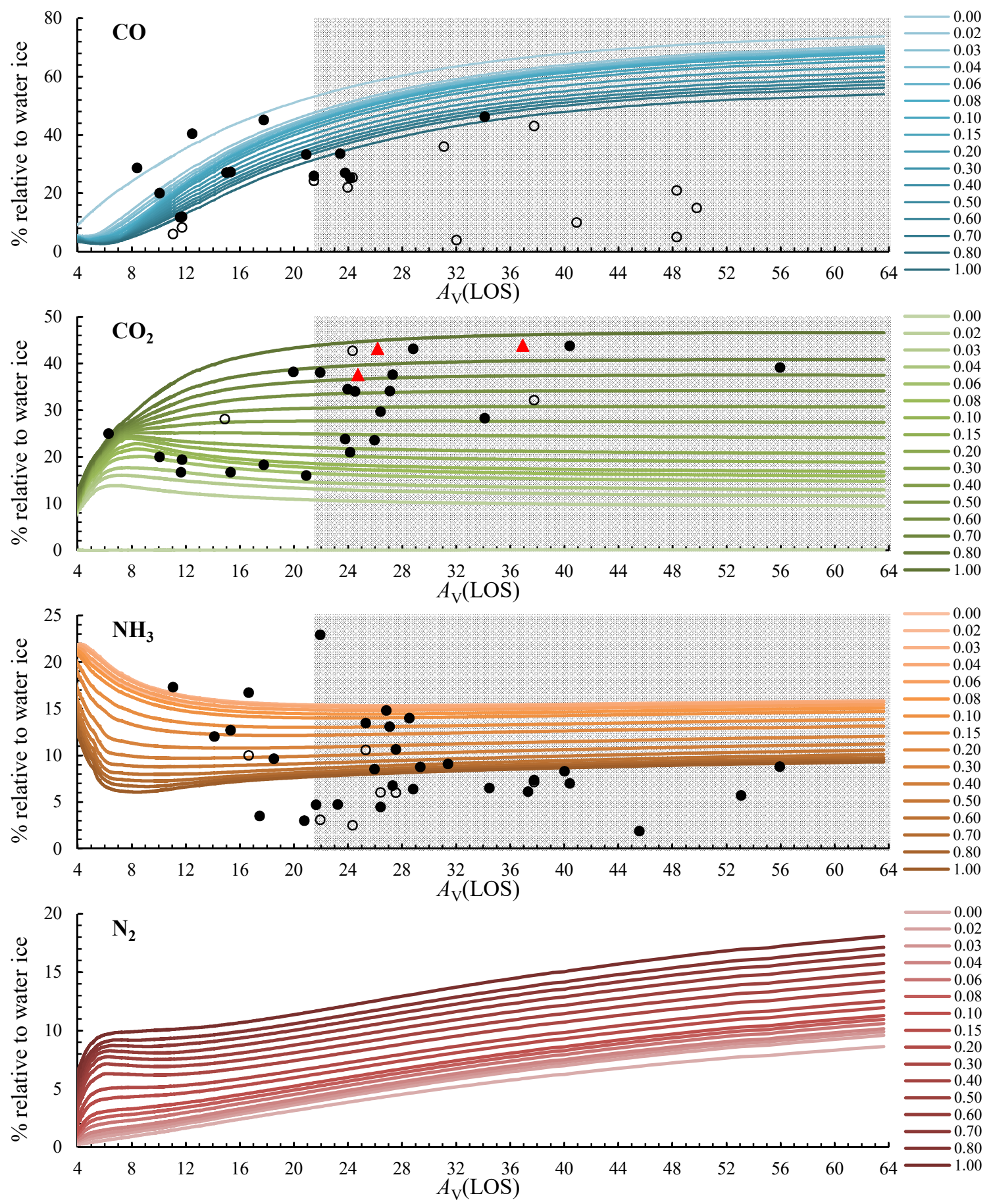

Figure 3. Modelled $X_{\text {calc }}$ for $\mathrm{CO}, \mathrm{CO}_{2}, \mathrm{NH}_{3}$, and $\mathrm{N}_{2}$ ices relative to water ice (curves; from top to bottom) for simulations with indicated values of $\epsilon_{\mathrm{ph}}$ as functions of total interstellar extinction along the line of sight. These are compared to the observed abundances $X_{\text {obs }}$, described as follows. Filled circles: 'valid' data points in the context of this study; empty circles: observations sampling highly irradiated molecular clouds, as indicated in Table A1 red triangles: observations attributed to long-lived starless cores. The shaded area indicates $A_{\mathrm{V}}(\mathrm{LOS})>22 \mathrm{mag}$, where observational data was found to significantly deviate from calculations (see Section 3.2). In the case of $\mathrm{CO} 2$, several of the empty circles are outside the scope of the ordinate. 
Table 4. Total difference between observed and calculated LOS abundances for $\mathrm{CO}, \mathrm{CO}_{2}$ and $\mathrm{NH}_{3}$ ices relative to water ice.

\begin{tabular}{cccc}
\hline \multicolumn{4}{c}{$\sum\left(X_{\text {obs }}-X_{\text {calc }}\right)$, percentage points } \\
$\epsilon_{\mathrm{ph}}$ & $\mathrm{CO}$ & $\mathrm{CO}_{2}$ & $\mathrm{NH}_{3}$ \\
\hline 0.00 & -183.1 & 208.2 & -50.6 \\
0.02 & -95.9 & 101.9 & -56.7 \\
0.03 & -79.2 & 80.0 & -56.5 \\
0.04 & -68.8 & 65.8 & -55.0 \\
0.06 & -56.9 & 48.2 & -50.7 \\
0.08 & -49.4 & 36.9 & -45.8 \\
0.10 & -44.8 & 29.6 & -40.6 \\
0.15 & -35.5 & 16.5 & -28.6 \\
0.20 & -26.0 & 5.4 & -18.5 \\
0.30 & -8.4 & -13.7 & -3.3 \\
0.40 & 7.9 & -31.5 & 7.9 \\
0.50 & 23.4 & -49.2 & 17.0 \\
0.60 & 37.1 & -67.7 & 24.1 \\
0.70 & 48.6 & -86.4 & 29.0 \\
0.80 & 59.6 & -105.4 & 32.4 \\
1.00 & 80.8 & -136.4 & 35.7 \\
\hline
\end{tabular}

fied here by the high abundance of methanol ice), where the ice has been exposed to irradiation probably for hundreds of kyr (Kalvāns 2015b).

The direct correlation between $\mathrm{CO}_{2}$ and photoprocessing also means that the inclusion of a full LOS with the $1 \mathrm{D}$ model increases the calculated $\mathrm{CO}_{2}: \mathrm{H}_{2} \mathrm{O}$ ratio. This is because ices in the outer part of the cloud core are more exposed to the ISRF. For example, at the end of simulation $\left(A_{\mathrm{V}}(\mathrm{LOS})=64 \mathrm{mag}\right)$ with $\epsilon_{\mathrm{ph}}$ taken to be $0.30, \mathrm{CO}_{2}: \mathrm{H}_{2} \mathrm{O}$ at the very centre of the core is 19 per cent, while for the whole LOS it is 24 per cent.

While $\mathrm{NH}_{3}$ ice is formed via surface reactions and $\mathrm{N}_{2}$ is simply accreted from the gas, their surface chemistry is intertwined. Because $\mathrm{N}_{2}$ has smaller cross-section for ISRFinduced photodissociation $\left(1.35 \times 10^{-18} \mathrm{~cm}^{2}\right.$ versus that of $7.05 \times 10^{-18} \mathrm{~cm}^{2}$ for $\mathrm{NH}_{3}$ ) and is $\mathrm{N}_{2}$ shielded in the gas phase, any overall increase of photodissociation rates favours $\mathrm{N}_{2}$. This is also manifested in Fig. 3, where the abundance of $\mathrm{N}_{2}$ ice grows and that of $\mathrm{NH}_{3}$ ice is reduced by increasing values of $\epsilon_{\mathrm{ph}}$. This is primarily because the photodestruction of $\mathrm{NH}_{3}$ ice on the surface means that the remaining radicals must undergo binary reactions to recombine (e.g., $\mathrm{NH}+\mathrm{H}$, $\mathrm{NH}_{2}+\mathrm{H}$ ) and in each such step some molecules are lost to the gas phase (where they are destroyed) because of reactive desorption. The observational data seemingly confirm these theoretical findings: cloud cores under increased irradiation tend to show lower relative abundances $X_{\mathrm{obs}}$ of $\mathrm{NH}_{3}$ ice (Fig. 3).

\subsection{Calculation of ice photodissociation efficiency}

Before finally deriving the value of $\epsilon_{\mathrm{ph}}$, we note that astrochemical models consider thousands of molecular processes with approximate rates, and a number of free parameters. The impact of uncertainties in these values can be significant and has been assessed in a number of studies (e.g., Vasvunin et al. 2004, 2008; Wakelam et al. 2005; Wakelam. Herbst \& Selsis 2006; Wakelam et al. 2010a b; Penteado, Walsh \& Cuppen 2017). In this study, we did not perform full sensitivity analysis of our model to the value of $\epsilon_{\mathrm{ph}}$. Instead, we attempted to identify the most likely value of $\epsilon_{\mathrm{ph}}$ by comparing the results of numerical simulations with observational data. A full analysis of the sensitivity of the modelling results to the parameters that control grainsurface chemistry is yet to be performed. Variations of some parameters $\left(E_{\mathrm{b}} / E_{\mathrm{D}}, E_{\mathrm{A}, \mathrm{O}+\mathrm{CO}}\right)$ that significantly affect surface chemistry were briefly investigated in order to identify a physically justified value that yields results, which agree to observations.

In order to acquire an estimate of $\epsilon_{\mathrm{ph}}$, we calculated the total differences $\sum\left(X_{\text {obs }}-X_{\text {calc }}\right)$ between observed $\left(X_{\text {obs }}\right)$ and calculated $\left(X_{\text {calc }}\right)$ relative abundances along LOS for icy species at corresponding $A_{\mathrm{V}}(\mathrm{LOS})$ values (i.e., the calculated and observed $\mathrm{CO}: \mathrm{H}_{2} \mathrm{O}, \mathrm{CO}_{2}: \mathrm{H}_{2} \mathrm{O}$, and $\mathrm{NH}_{3}: \mathrm{H}_{2} \mathrm{O}$ column density ratios). In this manner, we obtained a separate estimate of $\epsilon_{\mathrm{ph}}$ from each of the three icy species (carbon oxides and ammonia). This procedure was done only for the valid data points and in the interval of total interstellar extinction $0<A_{\mathrm{V}}(\mathrm{LOS}) \leqslant 22$ interval for calculations (Sections 3.1 and 3.2 . In Fig. 3, the data points used in this analysis can be identified as the black filled circles in the unshaded parts of the plots.

The three 'valid' observations of methanol ice were omitted from this analysis because of the high likelihood that, in most cases, the column densities of interstellar methanol ices towards background stars are too low to be detected, and thus the sample cannot be regarded as representative. According to the modelling results, a major ice component is molecular nitrogen; it could not be included in the analysis because of lack of observations. The number of the 'valid' observations was 12 for $\mathrm{CO}, 9$ for $\mathrm{CO}_{2}$ and 11 for $\mathrm{NH}_{3}$.

Table 4 shows that the total differences are the lowest for $\epsilon_{\mathrm{ph}}$ values $0.20,0.30$, and 0.40 . Therefore, we propose a value of 0.3 to be used as the ratio between solid and gas phase molecule photodissociation rate coefficients in astrochemical modelling.

\section{ACKNOWLEDGEMENTS}

This publication is supported by ERDF project Physical and chemical processes in the interstellar medium, No 1.1.1.1/16/A/213 being implemented in Ventspils University College. I thank Ventspils City Council for its financial support. I am grateful to Anton Vasyunin for a valuable discussion about this paper. This research has made use of NASA's Astrophysics Data System.

\section{REFERENCES}

Andersson S., Al-Halabi A., Kroes G.-J., van Dishoeck E. F., 2006, JChPh, 124, 064715

Andersson S., van Dishoeck E. F., 2008, A\&A, 491, 907

Arasa C., Koning J., Kroes G.-J., Walsh C., van Dishoeck E. F., 2015, A\&A, 575, A121

Bergin E. A., Langer W. D., Goldsmith P. F., 1995, ApJ, 441, 222

Bergin E. A., Melnick G. J., Gerakines P. A., Neufeld D. A., Whittet D. C. B., 2005, ApJL, 627, L33

Bertin M. et al., 2012, PCCP, 14, 9929 
Bertin M. et al., 2013, ApJ, 779, 120

Bertin M. et al., 2016, ApJL, 817, L12

Bonnor W. B., 1956, MNRAS, 116, 351

Boogert A. C. A., Chiar J. E., Knez C., Öberg K. I., Mundy

L. G., Pendleton Y. J., Tielens A. G. G. M., van Dishoeck

E. F., 2013, ApJ, 777, 73

Boogert A. C. A., Gerakines P. A., Whittet D. C. B., 2015, ARA\&A, 53, 541

Boogert A. C. A. et al., 2011, ApJ, 729, 92

Bossa J.-B., Paardekooper D. M., Isokoski K., Linnartz H., 2015,

Phys. Chem. Chem. Phys., 17, 17346

Bringa E. M., Johnson R. E., 2004, ApJ, 603, 159

Caselli P., Stantcheva T., Shalabiea O., Shematovich V. I., Herbst E., 2002, P\&SS, 50, 1257

Cazaux S., Martín-Doménech R., Chen Y. J., Muñoz Caro

G. M., González Díaz C., 2017, ApJ, 849, 80

Cecchi-Pestellini C., Aiello S., 1992, MNRAS, 258, 125

Chabot M., 2016, A\&A, 585, A15

Chang Q., Herbst E., 2016, ApJ, 819, 145

Chen Y.-J., Chuang K.-J., Muñoz Caro G. M., Nuevo M., Chu

C.-C., Yih T.-S., Ip W.-H., Wu C.-Y. R., 2014, ApJ, 781, 15

Chiar J. E. et al., 2011, ApJ, 731, 9

Chuang K.-J., Fedoseev G., Qasim D., Ioppolo S., van Dishoeck

E. F., Linnartz H., 2017, MNRAS, 467, 2552

Collings M. P., Anderson M. A., Chen R., Dever J. W., Viti S.,

Williams D. A., McCoustra M. R. S., 2004, MNRAS, 354, 1133

Cottin H., Moore M. H., Bénilan Y., 2003, ApJ, 590, 874

Cruz-Diaz G. A., Martín-Doménech R., Moreno E., Muñoz Caro

G. M., Chen Y.-J., 2018, MNRAS, 474, 3080

Cruz-Diaz G. A., Martín-Doménech R., Muñoz Caro G. M., Chen Y.-J., 2016, A\&A, 592, A68

Cruz-Diaz G. A., Muñoz Caro G. M., Chen Y.-J., Yih T.-S., 2014a, A\&A, 562, A119

Cruz-Diaz G. A., Muñoz Caro G. M., Chen Y.-J., Yih T.-S., 2014b, A\&A, 562, A120

Dartois E. et al., 2015, A\&A, 576, A125

D'Hendecourt L. B., Allamandola L. J., Greenberg J. M., 1985, A\&A, 152, 130

Dupuy R. et al., 2017, A\&A, 603, A61

Ebert R., 1955, ZA, 37, 217

Fayolle E. C., Balfe J., Loomis R., Bergner J., Graninger D.,

Rajappan M., Öberg K. I., 2016, ApJL, 816, L28

Fayolle E. C., Bertin M., Romanzin C., Michaut X., Öberg K. I.,

Linnartz H., Fillion J.-H., 2011b, ApJL, 739, L36

Fayolle E. C. et al., 2013, A\&A, 556, A122

Fedoseev G., Chuang K.-J., van Dishoeck E. F., Ioppolo S., Linnartz H., 2016, MNRAS, 460, 4297

Fillion J.-H. et al., 2014, FaDi, 168, 533

Furuya K., Aikawa Y., Nomura H., Hersant F., Wakelam V., 2013, ApJ, 779, 11

Furuya K., Drozdovskaya M. N., Visser R., van Dishoeck E. F., Walsh C., Harsono D., Hincelin U., Taquet V., 2017, A\&A, 599, A40

Garrod R. T., 2013, ApJ, 765, 60

Garrod R. T., Belloche A., Müller H. S. P., Menten K. M., 2017, A\&A, 601, A48

Garrod R. T., Herbst E., 2006, A\&A, 457, 927

Garrod R. T., Pauly T., 2011, ApJ, 735, 15

Garrod R. T., Vasyunin A. I., Semenov D. A., Wiebe D. S., Henning T., 2009, ApJL, 700, L43

Garrod R. T., Wakelam V., Herbst E., 2007, A\&A, 467, 1103

Garrod R. T., Weaver S. L. W., Herbst E., 2008, ApJ, 682, 283 Gerakines P. A., Moore M. H., Hudson R. L., 2004, Icarus, 170, 202

Gerakines P. A., Schutte W. A., Ehrenfreund P., 1996, A\&A, 312,289

Hasegawa T. I., Herbst E., 1993, MNRAS, 261, 83

Hasegawa T. I., Herbst E., Leung C. M., 1992, ApJS, 82, 167
Henderson B. L., Gudipati M. S., 2015, ApJ, 800, 66

Herbst E., Cuppen H. M., 2006, PNAS, 103, 12257

Hincelin U., Chang Q., Herbst E., 2015, A\&A, 574, A24

Hocuk S., Szűcs L., Caselli P., Cazaux S., Spaans M., Esplugues G. B., 2017, A\&A, 604, A58

Indriolo N., Fields B. D., McCall B. J., 2009, ApJ, 694, 257

Islam F., Baratta G. A., Palumbo M. E., 2014, A\&A, 561, A73

Ivlev A. V., Padovani M., Galli D., Caselli P., 2015, ApJ, 812, 135

Jiménez-Escobar A., Giuliano B. M., Muñoz Caro G. M., Cernicharo J., Marcelino N., 2014, ApJ, 788, 19

Johnson R. E., Donn B., Pirronello V., Sundqvist B., 1991, ApJL, 379, L75

Kalvāns J., 2015a, A\&A, 573, A38

Kalvāns J., 2015b, ApJ, 806, 196

Kalvāns J., 2015c, ApJ, 803, 52

Kalvāns J., 2016, ApJS, 224, 42

Kalvāns J., Shmeld I., 2010, A\&A, 521, A37

Kalvāns J., Shmeld I., Kalnin J. R., Hocuk S., 2017, MNRAS

Keto E., Caselli P., 2010, MNRAS, 402, 1625

Laas J. C., Garrod R. T., Herbst E., Widicus Weaver S. L., 2011, ApJ, 728, 71

Lee H.-H., Herbst E., Pineau des Forets G., Roueff E., Le Bourlot J., 1996, A\&A, 311, 690

Li X., Heays A. N., Visser R., Ubachs W., Lewis B. R., Gibson S. T., van Dishoeck E. F., 2013, A\&A, 555, A14

Lippok N. et al., 2013, A\&A, 560, A41

Luna R., Luna-Ferrándiz R., Millán C., Domingo M., Muñoz Caro G. M., Santonja C., Satorre M. Á., 2017, ApJ, 842, 51

Martín-Doménech R., Cruz-Díaz G. A., Muñoz Caro G. M., 2018, MNRAS, 473, 2575

Martín-Doménech R., Manzano-Santamaría J., Muñoz Caro G. M., Cruz-Díaz G. A., Chen Y.-J., Herrero V. J., Tanarro I., 2015, A\&A, 584, A14

Martín-Doménech R., Muñoz Caro G. M., Bueno J., Goesmann F., 2014, A\&A, 564, A8

Martín-Doménech R., Muñoz Caro G. M., Cruz-Díaz G. A., 2016, A\&A, 589, A107

Mason N. J. et al., 2006, FaDi, 133, 311

McCrea W. H., 1957, MNRAS, 117, 562

McElroy D., Walsh C., Markwick A. J., Cordiner M. A., Smith K., Millar T. J., 2013, A\&A, 550, A36

Minissale M., Congiu E., Manicò G., Pirronello V., Dulieu F., 2013, A\&A, 559, A49

Moskalenko I. V., Strong A. W., Ormes J. F., Potgieter M. S., 2002, ApJ, 565, 280

Muñoz Caro G. M., Chen Y.-J., Aparicio S., Jiménez-Escobar A., Rosu-Finsen A., Lasne J., McCoustra M. R. S., 2016, A\&A, 589, A19

Muñoz Caro G. M., Dartois E., Boduch P., Rothard H., Domaracka A., Jiménez-Escobar A., 2014, A\&A, 566, A93

Muñoz Caro G. M., Jiménez-Escobar A., Martín-Gago J. Á., Rogero C., Atienza C., Puertas S., Sobrado J. M., TorresRedondo J., 2010, A\&A, 522, A108

Nejad L. A. M., Williams D. A., Charnley S. B., 1990, MNRAS, 246,183

Öberg K. I., 2016, Chemical Reviews, 116, 9631, pMID: 27099922

Öberg K. I., Fuchs G. W., Awad Z., Fraser H. J., Schlemmer S., van Dishoeck E. F., Linnartz H., 2007, ApJL, 662, L23

Öberg K. I., Garrod R. T., van Dishoeck E. F., Linnartz H., 2009a, A\&A, 504, 891

Öberg K. I., Garrod R. T., van Dishoeck E. F., Linnartz H., 2011, in 2010 NASA Laboratory Astrophysics Workshop, p. C44

Öberg K. I., Linnartz H., Visser R., van Dishoeck E. F., 2009b, ApJ, 693, 1209 
Öberg K. I., van Dishoeck E. F., Linnartz H., 2009c, A\&A, 496, 281

Öberg K. I., van Dishoeck E. F., Linnartz H., Andersson S., 2010, ApJ, 718, 832

Paardekooper D. M., Bossa J.-B., Linnartz H., 2016, A\&A, 592, A67

Padovani M., Galli D., 2013, in ASSP, Vol. 34, Cosmic Rays in Star-Forming Environments, Torres D. F., Reimer O., eds., p. 61

Padovani M., Galli D., Glassgold A. E., 2009, A\&A, 501, 619

Penteado E. M., Walsh C., Cuppen H. M., 2017, ApJ, 844, 71

Plummer H. C., 1911, MNRAS, 71, 460

Roberts J. F., Rawlings J. M. C., Viti S., Williams D. A., 2007, MNRAS, 382, 733

Roser J. E., Vidali G., Manicò G., Pirronello V., 2001, ApJL, 555, L61

Ruaud M., Wakelam V., Hersant F., 2016, MNRAS, 459, 3756

Ruffle D. P., Herbst E., 2001a, MNRAS, 322, 770

Ruffle D. P., Herbst E., 2001b, MNRAS, 324, 1054

Semenov D. et al., 2010, A\&A, 522, A42

Shuping R. Y., Snow T. P., Chiar J. E., Kerr T., 2000, ApJ, 529, 932

Sipilä O., Caselli P., Taquet V., 2016, A\&A, 591, A9

Taquet V., Charnley S. B., Sipilä O., 2014, ApJ, 791, 1

Taquet V., Peters P. S., Kahane C., Ceccarelli C., LópezSepulcre A., Toubin C., Duflot D., Wiesenfeld L., 2013, A\&A, 550, A127

Teixeira T. C., Emerson J. P., 1999, A\&A, 351, 292

Thi W.-F., Woitke P., Kamp I., 2010, MNRAS, 407, 232

Tomasko M. G., Spitzer L., 1968, AJS, 73, 37

Vasyunin A. I., Caselli P., Dulieu F., Jiménez-Serra I., 2017, ApJ, 842, 33

Vasyunin A. I., Semenov D., Henning T., Wakelam V., Herbst E., Sobolev A. M., 2008, ApJ, 672, 629

Vasyunin A. I., Sobolev A. M., Wiebe D. S., Semenov D. A., 2004, AstL, 30, 566

Vaytet N., Haugbølle T., 2017, A\&A, 598, A116

Wakelam V., Herbst E., Le Bourlot J., Hersant F., Selsis F., Guilloteau S., 2010a, A\&A, 517, A21

Wakelam V., Herbst E., Selsis F., 2006, A\&A, 451, 551

Wakelam V., Selsis F., Herbst E., Caselli P., 2005, A\&A, 444, 883

Wakelam V. et al., 2010b, SSRv, 156, 13

Whittet D. C. B., Cook A. M., Herbst E., Chiar J. E., Shenoy S. S., 2011, ApJ, 742, 28

Whittet D. C. B., Gerakines P. A., Hough J. H., Shenoy S. S., 2001, ApJ, 547, 872

Whittet D. C. B. et al., 1998, ApJL, 498, L159

Whittet D. C. B., Poteet C. A., Chiar J. E., Pagani L., Bajaj V. M., Horne D., Shenoy S. S., Adamson A. J., 2013, ApJ, 774, 102

Whittet D. C. B., Shenoy S. S., Bergin E. A., Chiar J. E., Gerakines P. A., Gibb E. L., Melnick G. J., Neufeld D. A., 2007, ApJ, 655, 332

Whitworth A. P., Ward-Thompson D., 2001, ApJ, 547, 317

\section{APPENDIX A: OBSERVATIONAL DATA EMPLOYED FOR COMPARISON WITH OBSERVATIONS.}

Table A1 summarizes observations of interstellar ices, considered in this study.

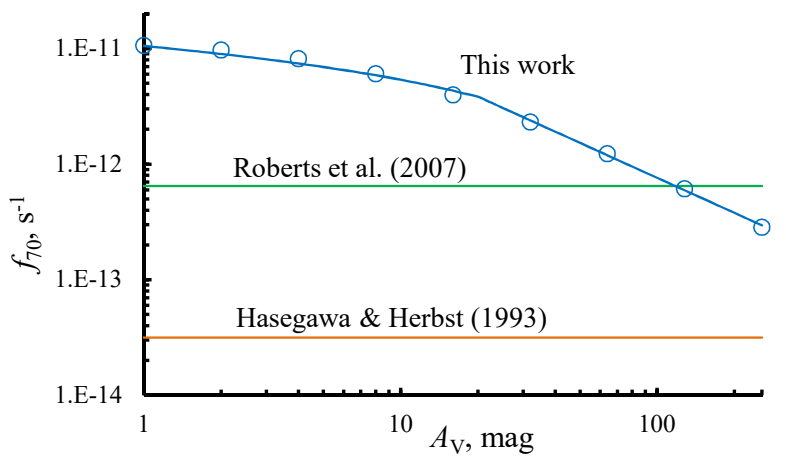

Figure B1. Calculated frequency of CR encounters with interstellar grains resulting in grain temperatures in excess of $70 \mathrm{~K}$. For comparison, the figure shows frequencies derived by previous studies.

\section{APPENDIX B: WHOLE-GRAIN HEATING FREQUENCY BY COSMIC RAYS.}

Our task is to obtain the frequency of CR encounters with interstellar grains that heat the grains to $70 \mathrm{~K}\left(f_{70}, \mathrm{~s}^{-1}\right)$ as a function of column density. For convenience, the latter is expressed in terms of interstellar extinction $A_{\mathrm{V}}=N_{\mathrm{H}} /\left(2.0 \times 10^{21}\right)$, where $N_{\mathrm{H}}\left(\mathrm{cm}^{-2}\right)$ is the total hydrogen column density from cloud edge to the modelled spatial point. From the grain types considered by Kalvāns (2016), we take $0.1 \mu \mathrm{m}$ grains with a thin $0.01 \mu \mathrm{m}$ ice layer at an equilibrium temperature of $10 \mathrm{~K}$. This is the type of grains most relevant to this study, where the accumulation of the icy mantles in a quiescent medium is considered. Kalvāns (2016) provides grain heating frequencies also for bare grains an grains with a $0.03 \mu \mathrm{m}$ thick ice layer.

The following procedure was used to acquire a reasonable estimate of $f_{70}$ as a function of $A_{\mathrm{V}}$. First, from the data of Kalvāns (2016) we identify that iron ions with an energy of $26 \mathrm{MeV} a m u^{-1}$ are the CR particles that contribute most to grain heating to a temperature of $70 \mathrm{~K}$. Second, with the methodology described by Chabot (2016) and Kalvāns (2016), we calculated the flux of these ions at $A_{\mathrm{V}}$ values equal to $2^{x}$ mag, where $x$ is $-1 ; 0 ; 1 ; 2$; $3 ; 4 ; 5 ; 6 ; 7$ and 8 (i.e., 0.5..256 mag). Third, we assumed that all the $\mathrm{CR}$ ions contributing to grain heating to $70 \mathrm{~K}$ are absorbed in the cloud in a manner similar to the $26 \mathrm{MeV}$ amu ${ }^{-1} \mathrm{Fe}$ ions. In other words, the $26 \mathrm{MeV}$ amu ${ }^{-1}$ Fe ions dominate grain heating to $70 \mathrm{~K}$. Thus, a set of source data points were obtained.

These points imply a rather high rate for whole-grain heating, corresponding to a CR spectrum with abundant low-energy $\left(<100 \mathrm{MeV} \mathrm{amu}^{-1}\right)$ particles (Moskalenko et al. 2002). However, two aspects limit the grain heating rate. First, it has been shown that this type of CR spectra has its intensity effectively reduced by relatively shallow hydrogen column densities (Padovani, Galli \& Glassgold 2009). For example, the intensity of all CR species is reduced by almost two orders of magnitude between $\mathrm{H}_{2}$ column densities of $10^{21} \mathrm{~cm}^{-2}$ and $10^{23} \mathrm{~cm}^{-2}$ (Chabot 2016). Because of this, it is necessary to adopt the same geometrical setting we employed for the ISRF, i.e., CRs are allowed to enter the cloud core only from two opposite sides of the cloud. This reduces the values of $f_{70}$ data points by a factor of $\approx 1 /(2 \pi)$ Kalvāns 2016, considered isotropic irradiation from all sides, i.e., spherical geometry). Second, similarly to the case of $\zeta$ (Section 2.1), we adopt a factor of 0.3 that modifies the CR flux because of magnetic reflection.

$A_{\mathrm{V}}$-dependent analytical function of $f_{70}$ was then obtained by interpolating the acquired data points. The grains are heated by CRs to a temperature of $70 \mathrm{~K}$ with the frequency $f_{70}$ for the interval $0.5 \leqslant A_{\mathrm{V}}<20$ :

$$
f_{70}=-2.246 \times 10^{-12} \ln \left(A_{\mathrm{V}}\right)+1.055 \times 10^{-11} ;
$$


Table A1. Observational data employed in the paper: interstellar extinction along LOS and respective relative abundances $X_{\mathrm{obs}}$ of icy species.

\begin{tabular}{|c|c|c|c|c|c|c|c|c|}
\hline Source ID & Other ID & $A_{\mathrm{V}}(\mathrm{LOS}), \mathrm{mag}$ & $\frac{\mathrm{CO}}{\mathrm{H}_{2} \mathrm{O}}, \%$ & $\frac{\mathrm{CO}_{2}}{\mathrm{H}_{2} \mathrm{O}}, \%$ & $\frac{\mathrm{NH}_{3}}{\mathrm{H}_{2} \mathrm{O}}, \%^{a}$ & $\frac{\mathrm{CH}_{3} \mathrm{OH}}{\mathrm{H}_{2} \mathrm{O}}, \%$ & Reference & Notes \\
\hline & Elias 3 & 10 & 20.0 & 20 & $\ldots$ & $\ldots$ & Bergin et al. (2005) & \\
\hline 181606000225539 & & 11.0 & $\ldots$ & . . & 17.29 & $\ldots$ & Boogert et al. (2011) & \\
\hline $21240614+4958310$ & & 14.1 & $\ldots$ & $\ldots$ & 12.01 & $\ldots$ & Boogert et al. (2011) & \\
\hline $22063773+5904520$ & & 15.3 & $\ldots$ & $\ldots$ & 12.70 & $\ldots$ & Boogert et al. (2011) & \\
\hline 080931353604035 & & 16.6 & $\ldots$ & $\ldots$ & 16.71 & $\ldots$ & Boogert et al. (2011) & \\
\hline 181407120708413 & & 16.6 & $\ldots$ & $\ldots$ & 9.99 & $\ldots$ & Boogert et al. (2011) & \\
\hline $19214480+1121203$ & & 18.5 & $\ldots$ & $\ldots$ & 9.65 & $\cdots$ & Boogert et al. (2011) & \\
\hline 171608602058142 & & 21.6 & $\ldots$ & $\ldots$ & 4.72 & $\ldots$ & Boogert et al. (2011) & \\
\hline 181704261802408 & & 22.0 & $\ldots$ & $\ldots$ & 22.90 & $\ldots$ & Boogert et al. (2011) & \\
\hline $19201622+1136292$ & & 22.0 & $\ldots$ & 38.08 & 3.09 & $\ldots$ & Boogert et al. (2011) & \\
\hline 171555732055312 & & 23.3 & $\ldots$ & $\ldots$ & 4.74 & $\ldots$ & Boogert et al. (2011) & \\
\hline 154216995247439 & & 25.3 & $\ldots$ & . & 13.45 & $\ldots$ & Boogert et al. (2011) & \\
\hline 181704291802540 & & 26.0 & $\ldots$ & 23.55 & 8.52 & $\ldots$ & Boogert et al. (2011) & \\
\hline 181652961801287 & & 26.4 & $\ldots$ & $\ldots$ & 4.48 & $\ldots$ & Boogert et al. (2011) & \\
\hline $04393886+2611266$ & & 26.4 & $\ldots$ & 29.66 & 6.03 & $\ldots$ & Boogert et al. (2011) & \\
\hline $04215402+1530299$ & & 26.8 & $\ldots$ & $\ldots$ & 14.80 & $\ldots$ & Boogert et al. (2011) & \\
\hline 120145986508586 & & 27.0 & $\ldots$ & 34.11 & 13.07 & $\ldots$ & Boogert et al. (2011) & \\
\hline $21240517+4959100$ & & 27.3 & $\ldots$ & 37.60 & 6.76 & $\ldots$ & Boogert et al. (2011) & \\
\hline $19201597+1135146$ & & 27.6 & $\ldots$ & $\ldots$ & 10.64 & $\ldots$ & Boogert et al. (2011) & \\
\hline 171604672057072 & & 28.5 & $\ldots$ & $\ldots$ & 13.99 & $\ldots$ & Boogert et al. (2011) & \\
\hline 181709570814136 & & 28.8 & $\ldots$ & 43.12 & 6.39 & $\ldots$ & Boogert et al. (2011) & \\
\hline 181659171801158 & & 29.4 & $\ldots$ & $\ldots$ & 8.76 & $\ldots$ & Boogert et al. (2011) & \\
\hline 181713660813188 & & 31.5 & $\ldots$ & $\ldots$ & 9.08 & $\ldots$ & Boogert et al. (2011) & \\
\hline 171115012726180 & & 34.4 & $\ldots$ & $\ldots$ & 6.49 & $\ldots$ & Boogert et al. (2011) & \\
\hline 181704700814495 & & 37.6 & $\ldots$ & $\ldots$ & 6.13 & $\ldots$ & Boogert et al. (2011) & \\
\hline 181711810814012 & & 37.9 & $\ldots$ & $\ldots$ & 7.35 & $\ldots$ & Boogert et al. (2011) & \\
\hline 080934683605266 & & 39.8 & $\ldots$ & $\ldots$ & 8.29 & $\ldots$ & Boogert et al. (2011) & \\
\hline 181726900438406 & & 40.5 & $\ldots$ & 43.75 & 7.01 & $\ldots$ & Boogert et al. (2011) & \\
\hline 120143016508422 & & 45.5 & $\ldots$ & $\ldots$ & 1.89 & $\ldots$ & Boogert et al. (2011) & \\
\hline 171120052727131 & & 52.8 & $\ldots$ & $\ldots$ & 5.71 & $\cdots$ & Boogert et al. (2011) & \\
\hline $18300061+0115201$ & & 56.1 & $\ldots$ & 39.14 & 8.79 & $\ldots$ & Boogert et al. (2011) & \\
\hline 160221284158478 & & 10.25 & $\ldots$ & 58.87 & $\ldots$ & $\ldots$ & Boogert et al. (2013) & $b$ \\
\hline 160211024158468 & & 11.05 & $\ldots$ & 187.8 & $\ldots$ & $\ldots$ & Boogert et al. (2013) & $b$ \\
\hline 160049254150320 & & 14.85 & $\ldots$ & 28.08 & $\ldots$ & $\ldots$ & Boogert et al. (2013) & $b$ \\
\hline 160128254153521 & & 24.25 & $\ldots$ & 80.32 & $\ldots$ & $\ldots$ & Boogert et al. (2013) & $b$ \\
\hline 154527473425184 & & 24.3 & 25.44 & 42.74 & 2.5 & $\ldots$ & Boogert et al. (2013) & $b$ \\
\hline 160126354150422 & & 25.35 & $\ldots$ & $\ldots$ & 10.58 & $\ldots$ & Boogert et al. (2013) & $b$ \\
\hline 154236993407362 & & 27.6 & $\ldots$ & $\ldots$ & 6.01 & $\ldots$ & Boogert et al. (2013) & $b$ \\
\hline 160106424202023 & & 29.45 & $\ldots$ & 99.8 & $\ldots$ & $\ldots$ & Boogert et al. (2013) & $b$ \\
\hline 160047394203573 & & 31.25 & $\ldots$ & 71.88 & $\ldots$ & $\ldots$ & Boogert et al. (2013) & $b$ \\
\hline 160142544153064 & & 37.8 & 43.1 & 32.14 & 7.14 & $\ldots$ & Boogert et al. (2013) & $b$ \\
\hline $21443293+4734569$ & Q22-1 & 17.49 & $\ldots$ & $\ldots$ & 3.5 & 1.1 & Chiar et al. (2011) & \\
\hline \multirow[t]{9}{*}{$21461164+4734542$} & Q21-6 & 20.7 & $\ldots$ & $\ldots$ & 3 & 1.6 & Chiar et al. (2011) & \\
\hline & Elias 25 & 11 & 6 & $\ldots$ & $\ldots$ & $\ldots$ & Shuping et al. (2000) & $c$ \\
\hline & Elias 32 & 24 & 22 & $\ldots$ & $\ldots$ & $\ldots$ & Shuping et al. (2000) & $c$ \\
\hline & Elias 33 & 31 & 36 & $\ldots$ & $\ldots$ & $\ldots$ & Shuping et al. (2000) & $c$ \\
\hline & WL 16 & 32 & 4 & $\ldots$ & $\ldots$ & $\ldots$ & Shuping et al. (2000) & $c$ \\
\hline & WL 12 & 41 & 10 & $\ldots$ & $\ldots$ & $\ldots$ & Shuping et al. (2000) & $c$ \\
\hline & Elias 29 & 48 & 5 & $\ldots$ & $\cdots$ & . . & Shuping et al. (2000) & $c$ \\
\hline & WL 6 & 48 & 21 & $\ldots$ & $\ldots$ & . . & Shuping et al. (2000) & $c$ \\
\hline & WL 5 & 50 & 15 & $\ldots$ & $\ldots$ & $\ldots$ & Shuping et al. (2000) & $c$ \\
\hline $042324.6+250009$ & Elias 3 & 8.4 & 28.7 & $\ldots$ & $\ldots$ & $\ldots$ & Teixeira \& Emerson (1999) & \\
\hline $043325.9+261534$ & Elias 13 & 11.6 & 11.8 & $\ldots$ & $\ldots$ & $\ldots$ & Teixeira \& Emerson (1999) & \\
\hline $043926.8+255259$ & Elias 15 & 15 & 27.1 & $\ldots$ & $\ldots$ & $\ldots$ & Teixeira \& Emerson (1999) & \\
\hline $044058.1+255416$ & Tamura 8 & 21.5 & 26.0 & $\ldots$ & $\ldots$ & $\cdots$ & Teixeira \& Emerson (1999) & \\
\hline $043938.7+261125$ & Elias 16 & 23.5 & 33.6 & $\ldots$ & . . & . . & Teixeira \& Emerson (1999) & \\
\hline $043728.2+261024$ & Tamura 2 & 6.3 & $\ldots$ & 25.0 & $\ldots$ & $\ldots$ & Whittet et al. (2007) & \\
\hline $043325.9+261534$ & Elias 13 & 11.7 & 12.0 & 19.4 & $\ldots$ & . . & Whittet et al. (2007) & \\
\hline $043926.9+255259$ & Elias 15 & 15.3 & 27.3 & 16.7 & $\ldots$ & $\ldots$ & Whittet et al. (2007) & \\
\hline $042630.7+243637$ & & 17.8 & 45.1 & 18.3 & $\ldots$ & $\ldots$ & Whittet et al. (2007) & \\
\hline $043213.2+242910$ & & 20.9 & 33.3 & 16.0 & $\ldots$ & $\ldots$ & Whittet et al. (2007) & \\
\hline $044057.5+255413$ & Tamura 8 & 21.5 & 24.3 & $\ldots$ & $\ldots$ & $\ldots$ & Whittet et al. (2007) & \\
\hline $043938.9+261125$ & Elias 16 & 24.1 & 25.3 & 21.0 & $\ldots$ & $\ldots$ & Whittet et al. (2007) & \\
\hline
\end{tabular}


Table A2. Table A1 continued.

\begin{tabular}{|c|c|c|c|c|c|c|c|c|}
\hline Source ID & Other ID & $A_{\mathrm{V}}(\mathrm{LOS}), \mathrm{mag}$ & $\frac{\mathrm{CO}}{\mathrm{H}_{2} \mathrm{O}}, \%$ & $\frac{\mathrm{CO}_{2}}{\mathrm{H}_{2} \mathrm{O}}, \%$ & $\frac{\mathrm{NH}_{3}}{\mathrm{H}_{2} \mathrm{O}}, \%^{a}$ & $\frac{\mathrm{CH}_{3} \mathrm{OH}}{\mathrm{H}_{2} \mathrm{O}}, \%$ & Reference & Notes \\
\hline J04332594+2615334 & Elias 13 & 11.7 & 8.3 & 16.7 & $\ldots$ & $\ldots$ & Whittet et al. (2011) & \\
\hline J181407120708413 & & 15.1 & . & $\ldots$ & $\ldots$ & 5.3 & Whittet et al. (2011) & $d$ \\
\hline J19201622+1136292 & & 20.0 & . & 38.2 & $\ldots$ & $\ldots$ & Whittet et al. (2011) & \\
\hline J181704291802540 & & 23.7 & . & 23.8 & $\ldots$ & $\ldots$ & Whittet et al. (2011) & \\
\hline $\mathrm{J} 21472204+4734410$ & Q21-1 & 23.9 & 27.0 & 34.5 & $\ldots$ & 0.9 & Whittet et al. (2011) & \\
\hline J120145986508586 & & 24.6 & . & 34.0 & $\ldots$ & & Whittet et al. (2011) & \\
\hline $\mathrm{J} 21240517+4959100$ & & 24.8 & . & 37.6 & $\ldots$ & 7.0 & Whittet et al. (2011) & $d$ \\
\hline J181709570814136 & & 26.2 & . & 43.2 & $\ldots$ & 10.0 & Whittet et al. (2011) & $d$ \\
\hline J181713660813188 & & 28.6 & . & $\ldots$ & $\ldots$ & 10.9 & Whittet et al. (2011) & $d$ \\
\hline $\mathrm{J} 18300061+0115201$ & CK2, EC 118 & 34 & 46.3 & 28.3 & $\ldots$ & $\ldots$ & Whittet et al. (2011) & \\
\hline J181704700814495 & & 34.2 & . & $\ldots$ & $\ldots$ & 6.8 & Whittet et al. (2011) & $d$ \\
\hline J181711810814012 & & 34.5 & . & $\ldots$ & $\ldots$ & 12.3 & Whittet et al. (2011) & $d$ \\
\hline J181726900438406 & & 36.8 & . & 43.9 & $\ldots$ & 8.9 & Whittet et al. (2011) & $d$ \\
\hline J155420440254073 & & 12.5 & 40.4 & & $\ldots$ & $\ldots$ & Whittet et al. (2013) & \\
\hline
\end{tabular}

${ }^{a}$ Includes $\mathrm{NH}_{4}^{+} \cdot{ }^{b}$ Lupus cloud; increased irradiation. ${ }^{c} \rho$ Oph cloud; strong irradiation. ${ }^{d}$ Identified as long-lived stable starless cloud core.

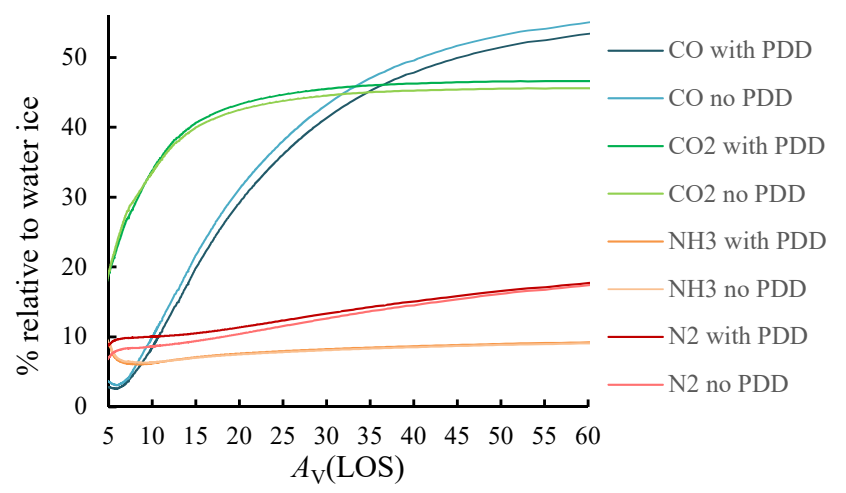

Figure C1. Comparison of calculated relative abundances of abundant icy species for a model with and without photodissociative desorption (see text). For maximum effect, photodissociation efficiency $\epsilon_{\mathrm{ph}}$ was taken to be 1.0.

and for $20 \leqslant A_{\mathrm{V}} \leqslant 256$ mag:

$$
f_{70}=7.826 \times 10^{-11} A_{\mathrm{V}}^{-1.006} .
$$

The resulting function is shown graphically in Figure B1 To calculate the desorption rate for species $i, f_{70}$ must be used according to Equation (3) in the paper.

\section{APPENDIX C: MODEL FOR PHOTODISSOCIATIVE DESORPTION.}

Dissociation of surface water results in gas-phase products containing the oxygen atom (i.e., PDD) with a rate that is equal to 3 per cent of the total dissociation rate Andersson \& van Dishoeck 2008). From this we assume that 3 percent of all photodissociated surface species are desorbed in the model. The photodissociation coefficient (total) was calculated with Equation (4) with $B=0$. As a rule, the PDD rate never exceeds the total photodesorption rate. This means that the photodesorption rate (coefficient calculated with Equation (5) accounts for both, the desorption of intact and dissociated species. This approach on PDD is rather conservative; however, it is an improvement over our previous models that did not consider PDD at all.

The adopted approach results in that $\mathrm{CO}$ and $\mathrm{N}_{2}$ are desorbed intact for most of the time, while other impor- tant species are largely removed via $\mathrm{PDD}$, which is in general agreement with experiments (e.g., Öberg et al. 2009b; Öberg, van Dishoeck \& Linnartz 2009c; Favolle et al. 2011b, 2013; Bertin et al. 2012, 2016; Fillion et al. 2014; Cruz-Diaz et al. 2016). Because the dissociation rate coefficients tend to decrease more rapidly with $A_{\mathrm{V}}$, the effects of PDD diminish with cloud evolution in our model. For example, at $A_{\mathrm{V}}(L O S)=5 \mathrm{mag}, 99.8$ per cent of $\mathrm{CO}$ and 50.4 per cent of $\mathrm{H}_{2} \mathrm{O}$ molecules are desorbed intact by ISRF photons. At $A_{\mathrm{V}}(L O S)=50 \mathrm{mag}$, these numbers are 100.0 and 98.9 , respectively. Therefore, the PDD rates do not become overestimated. Figure C1 shows that the effects of PDD on the relative abundances of major icy species are relatively negligible for this study. They are even less pronounced for simulations with lower values of $\epsilon_{\mathrm{ph}}$ (i.e., lower icy molecule photodissociation rates).

The rate for water photodissociation by the ISRF at $A_{\mathrm{V}}$ values below $2 \mathrm{mag}$ can be close to $10^{-16} \mathrm{~s}^{-1} \mathrm{~cm}^{-3}$. This is comparable to the rate of free $\mathrm{H}$ atoms sticking on grain surfaces. Therefore, the photolysis of hydrogen-containing molecules might serve as an important source of atomic hydrogen on the surface. However, this is likely not the case. Most of $\mathrm{H}$ atoms, split-off from surface molecules, leave the grain (Andersson et al. 2006). To reflect this aspect in the model, we assumed that 95 per cent of all $\mathrm{H}$ atoms (and $\mathrm{H}_{2}$ molecules) split off from surface $\mathrm{H}_{2} \mathrm{O}$ are desorbed (Arasa et al. 2015). This rule was attributed also to the two other major hydrogenated surface species ammonia $\mathrm{NH}_{3}$ and methanol $\mathrm{CH}_{3} \mathrm{OH}$. 